\title{
Nipah virus outbreak with person-to-person transmission in a district of Bangladesh, 2007
}

\author{
N. HOMAIRA ${ }^{1,2 *}$, M. RAHMAN ${ }^{1}$, M. J. HOSSAIN ${ }^{2}$, J. H. EPSTEIN ${ }^{3,4}$, \\ R. SULTANA ${ }^{2}$, M. S. U. KHAN ${ }^{2}$, G. PODDER ${ }^{2}$, K. NAHAR ${ }^{2}$, B. AHMED ${ }^{1}$, \\ E. S. GURLEY ${ }^{2}$, P. DASZAK ${ }^{4}$, W. I. LIPKIN ${ }^{5}$, P. E. ROLLIN ${ }^{6}$, J. A. COMER ${ }^{6}$, \\ T. G. KSIAZEK ${ }^{6,7}$ AND S. P. LUBY ${ }^{2,6}$ \\ ${ }^{1}$ Institute of Epidemiology, Disease Control and Research (IEDCR) Dhaka, Bangladesh \\ ${ }^{2}$ International Centre for Diarrhoeal Disease Research, Bangladesh (ICDDR,B), Dhaka, Bangladesh \\ ${ }^{3}$ The Consortium for Conservation Medicine (CCM), New York, USA \\ ${ }^{4}$ Wildlife Trust, New York, NY USA \\ ${ }^{5}$ Center for Infection and Immunity, Columbia University, New York, USA \\ ${ }^{6}$ Special Pathogens Branch, Division of Viral and Rickettsial Disease, National Centre for Infectious Disease, \\ Centers for Disease Control and Prevention (CDC), Atlanta, GA, USA \\ ${ }^{7}$ Galveston National Laboratory, Department of Pathology, Galveston TX, USA
}

(Accepted 9 March 2010)

\section{SUMMARY}

In February 2007 an outbreak of Nipah virus (NiV) encephalitis in Thakurgaon District of northwest Bangladesh affected seven people, three of whom died. All subsequent cases developed illness 7-14 days after close physical contact with the index case while he was ill. Cases were more likely than controls to have been in the same room (100\%vs. $9 \cdot 5 \%$, OR undefined, $P<0.001)$ and to have touched him $(83 \%$ vs. $0 \%$, OR undefined, $P<0.001)$. Although the source of infection for the index case was not identified, $50 \%$ of Pteropus bats sampled from near the outbreak area 1 month after the outbreak had antibodies to NiV confirming the presence of the virus in the area. The outbreak was spread by person-to-person transmission. Risk of $\mathrm{NiV}$ infection in family caregivers highlights the need for infection control practices to limit transmission of potentially infectious body secretions.

Key words: Bangladesh, Nipah virus, person-to-person transmission.

\section{INTRODUCTION}

In Bangladesh, Nipah virus (NiV) was first identified as the cause of an outbreak of encephalitis in 2001 in Meherpur District [1, 2]. Four additional outbreaks were identified between 2001 and 2005 [1-4].

\footnotetext{
* Author for correspondence: Dr N. Homaira, Programme on Infectious Disease and Vaccine Sciences, Health System and Infectious Disease Division, ICDDR,B, 68, Shahid Tajuddin Ahmed Sharani, Mohakhali, Dhaka-1212, Bangladesh. (Email: nhomaira@icddrb.org)
}

Antibodies reactive to $\mathrm{NiV}$ antigen have been detected in pteropid bats in both India and Bangladesh $[1,5]$.

Person-to-person transmission of $\mathrm{NiV}$ infection, following human infection directly from the environment, was noted in previous outbreaks in the Indian subcontinent. In a NiV outbreak in Siliguri, India in 2001,45 patients $(75 \%)$ had a history of hospital exposure to other patients with $\mathrm{NiV}$ infection [6]. In Faridpur District, Bangladesh in 2004 NiV casepatients in Faridpur were seven times more likely than 
non-patients to have had close contact with one of the $\mathrm{NiV}$ patients [odds ratio (OR) 6.7, 95\% confidence interval (CI) 2·9-16.8, $P<0 \cdot 001]$ [2].

On 9 February 2007, a physician at Rangpur Medical College Hospital, one of 10 hospitals involved in active NiV encephalitis surveillance in Bangladesh, reported a cluster of fatal encephalitis involving a husband and a wife residing in the Haripur Upazila (subdistrict) of Thakurgaon District. Both patients had similar symptoms and died within an interval of 2 weeks. A collaborative team including the Institute of Epidemiology Disease Control and Research (IEDCR) and the International Centre for Diarrhoeal Disease Research, Bangladesh (ICDDR,B), began an investigation on 10 February 2007. The objectives of the investigation were to identify the cause of the outbreak and the risk factors for developing illness.

\section{METHODS}

\section{Case definition and identification}

We defined suspected case-patients as persons having fever with altered mental status or new onset of seizures (severe illness), or persons having fever with headache or cough (mild illness), residing in the outbreak area with an onset of illness between 15 January and 28 February 2007. The team visited the outbreak village and asked the community health workers and community residents if they were aware of any patient who was suffering from fever with seizure or altered mental status, or who had died from these symptoms in their neighbourhood. We also asked them about case-patients suffering from fever with headache and/ or cough. The team then visited the local hospital in order to identify suspected case-patients. Team members also investigated all the deaths in the outbreak village between January and February. We obtained a history of illness and general information about exposures for each suspected case-patient. We asked the local health authority of the affected subdistrict to report to the IEDCR if they identified any further suspected case-patient having fever and altered mental status or seizures who sought treatment in the local subdistrict health complex during February.

The team collected blood samples from living suspected case-patients, which were centrifuged in the field and transported on wet ice to IEDCR, where they were stored at $-70^{\circ}$. Samples were tested with an immunoglobulin $\mathrm{M}$ ( $\operatorname{IgM})$ capture enzyme-linked immunosorbent assay (ELISA) that detects IgM antibodies specific for NiV antigens [7].

We defined a confirmed case of NiV infection as a suspected case-patient with detectable IgM to NiV. The team defined a probable NiV case-patient as a patient with fever and altered mental status who lived in the same village as a confirmed case-patient during the outbreak period, but from whom serum was not available because the patient died before a specimen could be collected.

\section{Qualitative study}

A team of experienced anthropologists conducted in-depth interviews and informal discussions with available confirmed and probable case-patients, their family members and friends, and other residents in these communities with the goals of exploring potential exposures to $\mathrm{NiV}$ and identifying appropriate proxy respondents for deceased cases or cases that were too sick to interview. The anthropologists also collected information about symptoms of the disease, caregiving practices and health facility utilization by persons affected by the outbreak.

\section{Case-control study}

We conducted a case-control study to investigate exposures associated with $\mathrm{NiV}$ infection, including person-to-person transmission. Probable and confirmed case-patients were enrolled as cases. We selected three controls for each case-patient. Controls were selected starting from the fourth closest house to the case-patient where no members were ill during the outbreak. The household resident closest in age to the case-patient was eligible to participate as a control. Participation was voluntary. If the selected household resident declined to participate, a resident from the next closest house was asked to participate.

The qualitative team selected proxy respondents for each case-patient who had died or was unable to respond. The proxy respondents included family members and friends of the case-patients who were most knowledgeable about their activities and probable risk exposures in the preceding 1 month before illness. Multiple proxy respondents were common. The investigation team used a standardized questionnaire to collect information on demographics, symptoms of illness, and possible risk factors associated with NiV transmission including history of consumption of date palm juice prior to illness, exposure 
to animals and exposure to ill patients, including touching, staying in the same room, feeding, sharing a bed or cleaning body secretions of a NiV patient.

\section{Bat survey}

A team of veterinarians from ICDDR,B with assistance from the Consortium for Conservation Medicine located two bat roosts which were $1 \mathrm{~km}$ and $15 \mathrm{~km}$ distant from the outbreak village. Bats were captured using mist nets and were anaesthetized during sample collection and released at the point of capture after sampling from 24 February to 9 March 2007. All the captured bats from which blood samples were collected were $P$. giganteus.

All bat blood samples were kept on ice until the end of each day when serum was separated and stored in liquid nitrogen. At the end of each day, blood samples were transferred to liquid nitrogen and transported to ICDDR,B where they were stored at $-70{ }^{\circ} \mathrm{C}$ and then shipped on dry ice to the Australian Animal Health Laboratory for laboratory diagnosis. All the blood samples were assayed for antibodies against NiV using a serum neutralization test.

\section{Statistics}

We analysed socio-demographic and clinical profiles of the case-patients using descriptive statistics. For the case-control study, we used ORs to estimate the association of each exposure with disease and calculated $95 \%$ CIs around the ORs. We used the $\chi^{2}$ test when expected cell sizes were $>5$ and Fisher's exact test when expected cell sizes were $<5$ and considered association to be statistically significant if the $P$ value was $<0 \cdot 05$. We used an unmatched analysis because neighbours were chosen as controls to ensure that controls and case-patients were representative of the same population and not to control for confounding factors. Because the primary hypothesis was that the index case was the source of $\mathrm{NiV}$ transmission for the subsequent cases, we excluded the index case, but none of the controls in the analyses of person-toperson transmission.

\section{Ethics}

All human study participants gave informed consent for participation in this investigation. The Ethical Review Committee at ICDDR,B reviewed and approved a protocol for encephalitis surveillance and outbreak investigation. Bat capture and sample collection was conducted under a protocol approved by the Institutional Animal Care and Use Committee.

\section{RESULTS}

\section{Descriptive epidemiology}

Eleven serum samples were collected from 13 suspected case-patients. Five suspected case-patients had IgM antibodies against $\mathrm{NiV}$ by capture ELISA and were thus confirmed cases. Two suspected casepatients had fever and altered mental status, but died before samples could be collected and were categorized as probable cases. These two probable cases were the index case and his wife. The remainder of the analysis was performed on these seven confirmed or probable case-patients. Five of these case-patients (three confirmed and two probable) had fever with altered mental status and three $(60 \%)$ of them died. A total of five case-patients, including the two probable cases, were hospitalized. The mean age of casepatients was 24 years (range 19-30 years) and five $(71 \%)$ were male. The median duration from onset of fever to death was 5.6 days (range 5-7) (Table 1). Fever $(100 \%)$, altered consciousness (71\%) along with vomiting $(71 \%)$ and cough $(71 \%)$ were the most common symptoms (Table 1).

\section{Qualitative findings}

The index case first developed fever on 21 January 2007 which progressed to headache, cough, breathing difficulties, convulsions, loss of consciousness and finally death 5 days later. In total 14 people who were family members, relatives or friends had physical contact with the index case when he was ill; six (43\%) of them developed $\mathrm{NiV}$ infection. Five of the subsequent cases had contact with the index case only during the last 2 days of his illness (incubation period 7-14 days). The dates of illness onset for subsequent cases ranged from 2 to 8 February 2007 (Fig. 1). None of the caregivers of the subsequent cases developed illness.

During the first 4 days of illness the index case was cared for at home, primarily by his wife and sisterin-law. They fed him, cleaned him and wiped froth and saliva from his mouth. They also massaged oil on his head and body to relieve him of pain. His wife shared the same bed with him and provided care throughout his illness. She became severely ill 14 days 


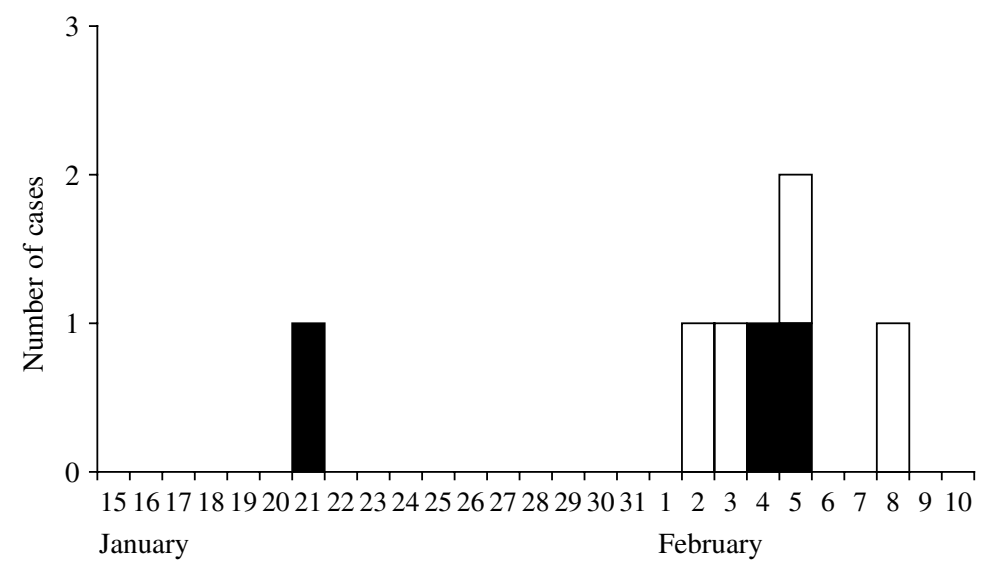

Fig. 1. Distribution of NiV cases by date of onset of illness, Haripur Upazila (subdistrict), Thakurgaon District, Bangladesh, January-February 2007. $\square$, Alive; $\mathbf{\square}$, died.

Table 1. Characteristics of case-patients, Haripur Upazila, Thakurgaon District, Bangladesh, February 2007

\begin{tabular}{lc}
\hline \hline Characteristics & $n=7(\%)$ \\
\hline Age & \\
Mean (years) & 24 \\
Median (range) & $24(19-30)$ \\
Male & $5(71)$ \\
Occupation & \\
Student & $2(29)$ \\
Housewife & $1(14)$ \\
Businessman & $2(29)$ \\
Driver & $2(29)$ \\
Clinical feature & \\
Fever & $7(100)$ \\
Severe fatigue/weakness & $6(86)$ \\
Headache & $3(43)$ \\
Vomiting & $5(71)$ \\
Cough & $5(71)$ \\
Respiratory distress & $4(57)$ \\
Altered mental status & $5(71)$ \\
Muscle pain & $4(57)$ \\
Restlessness & $4(57)$ \\
Unconscious & $2(29)$ \\
Joint pain & $1(14)$ \\
Case fatality & $3(43)$ \\
Onset of illness to death $(n=3)$, & $5 \cdot 6(5-7)$ \\
mean (range) & \\
\hline \hline &
\end{tabular}

after the husband's illness began and died within 6 days of illness.

A day before his death, the index case developed a severe cough and breathing difficulty. He was taken to a local doctor accompanied by a friend and a cousin. A chest radiograph of the index case taken

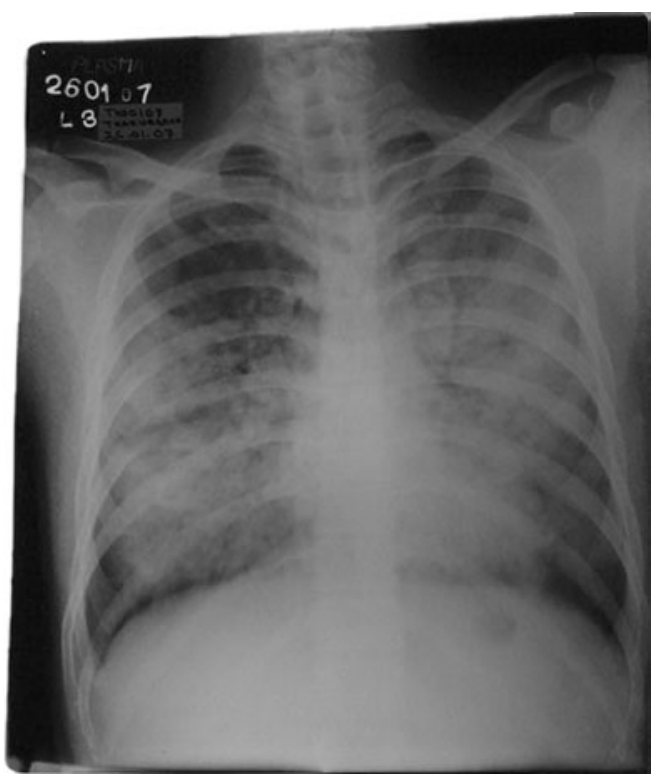

Fig. 2. Chest X-ray of the index case showing features of acute respiratory distress syndrome.

during this period showed diffuse bilateral opacity in both lung fields suggesting features of acute respiratory distress syndrome (Fig. 2). The friend became ill 11 days later and died after 7 days. The cousin also became ill 14 days after his physical contact with the index case.

The day after his chest radiograph, the index case developed reduced level of consciousness, and was admitted to a hospital where he died on the same day. The driver of a micro bus who helped transport and carry him to the hospital developed $\mathrm{NiV}$ infection 10 days after exposure.

While the index case was hospitalized, his wife's sister and one of his friends visited him in the hospital 
Table 2. Bivariate analysis of risk factors for Nipah virus infection, Haripur Upazila, Thakurgaon District, Bangladesh, February 2007

\begin{tabular}{|c|c|c|c|c|c|}
\hline Risk factors & $\begin{array}{l}\text { No. }(\%) \text { of } \\
\text { cases with } \\
\text { this risk factor }\end{array}$ & $\begin{array}{l}\text { No. }(\%) \text { of } \\
\text { controls with } \\
\text { this risk factor }\end{array}$ & OR & $95 \% \mathrm{CI}$ & $P$ value \\
\hline Male sex & $4(67 \%)$ & $8(38 \%)$ & $3 \cdot 25$ & $0 \cdot 48-22$ & $0 \cdot 2$ \\
\hline Climbed trees & $1(17)$ & $4(19 \%)$ & $0 \cdot 85$ & $0 \cdot 07-9 \cdot 4$ & $1 \cdot 00$ \\
\hline \multicolumn{6}{|l|}{ Physical contact with living animal } \\
\hline Pig & $0(0 \%)$ & $0(\%)$ & Undefined & & \\
\hline Fruit bat & $0(0 \%)$ & $0(0 \%)$ & Undefined & & \\
\hline Cow & $4(67 \%)$ & $13(62 \%)$ & $1 \cdot 23$ & $0 \cdot 182-8 \cdot 33$ & $1 \cdot 00$ \\
\hline Goat & $2(33 \%)$ & $9(43 \%)$ & $0 \cdot 67$ & $0 \cdot 099-4 \cdot 5$ & $1 \cdot 00$ \\
\hline Ate any animal that had been sick & $0(0 \%)$ & $1(5 \%)$ & Undefined & & $1 \cdot 00$ \\
\hline Drank raw date palm sap & $1(17 \%)$ & $0(0 \%)$ & Undefined & & $0 \cdot 22$ \\
\hline Visited the index case in a hospital & $6(100 \%)$ & $0(0 \%)$ & Undefined & & $<0.001$ \\
\hline Touched the index case when he was sick & $5(83 \cdot 3 \%)$ & $0(0 \%)$ & Undefined & & $<0.001$ \\
\hline $\begin{array}{l}\text { Been present in the same room with the index } \\
\text { case when he was sick }\end{array}$ & $6(100 \%)$ & $2(9 \cdot 5 \%)$ & Undefined & & $<0.001$ \\
\hline $\begin{array}{l}\text { Been present in the same room with the index } \\
\text { case when he was coughing }\end{array}$ & $6(100 \%)$ & $0(0 \%)$ & Undefined & & $0 \cdot 04$ \\
\hline
\end{tabular}

OR, Odds ratio; CI, confidence interval.

and fed and touched him. Both of them developed $\mathrm{NiV}$ infection within 10 days of contact.

\section{Case-control study}

We used proxy interviews for the three dead casepatients, but not for any controls. The mean age for cases and controls was similar [mean age $( \pm$ s.D. $)$ $24 \pm 4$ years in cases vs. $24 \pm 7$ years in controls, $t=-0 \cdot 097, P=0 \cdot 9]$. Cases were more likely to be males than controls but this could be due to chance (67\% males in case-patient group vs. $38 \%$ in control group, OR $3 \cdot 2,95 \%$ CI $0 \cdot 5-22, P=0 \cdot 2$ ).

$\mathrm{NiV}$ case-patients were more likely than controls to have consumed raw date palm sap in the 15 days prior to illness ( $29 \%$ in case-patients $v s .0 \%$ in controls, OR undefined, $P=0.056)$. Two $(29 \%)$ of the case-patients including the index case who had consumed raw date palm juice bought it from a vendor in the local village market. Although there were sick goats in the outbreak-affected community, none of the cases or controls had any contact with them or any other sick animal within 15 days prior to illness. Moreover, there was no report of contact with fruit bats.

In the analysis for person-to-person transmission, case-patients were more likely than controls to have been present in the same room with $(100 \%$ vs. $9 \cdot 5 \%$, OR undefined, $P=0.000)$ or touched $(83 \%$ vs. $0 \%$,
OR undefined, $P=0 \cdot 000$ ) the index case. In a subanalysis in those who stayed in the same room with the index case, case-patients were more likely than controls to be present in the same room when he was coughing ( $100 \%$ vs. $0 \%$, OR undefined, $P=0.04)$. Only case-patients had hospital exposure to other $\mathrm{NiV}$ case-patients $(86 \%$ vs. $0 \%, P=0.000)$, with all reporting visits to the index case in the hospital (Table 2).

\section{Bat study}

The team captured and sampled $118 P$. giganteus bats; 29 of which were juvenile bats. Of the 67 bats sampled $1 \mathrm{~km}$ from the outbreak village, $34(51 \%)$ tested positive on serum neutralization test [median titre 30 , range 5 to $>640$ ]. Three of the 34 serum neutralization test-positive bats had $\mathrm{NiV}$ antibody titres $>640$. In the neighbouring village, $15 \mathrm{~km}$ away, $27 / 51$ bats $(53 \%)$ had serum neutralizing antibodies to $\mathrm{NiV}$ [median titre 20, range 5-320]. Of the 61 pteropid bats that were seropositive seven were juvenile bats [median titre 15, range 5-20].

\section{DISCUSSION}

Several lines of evidence suggest person-to-person transmission as the primary route of transmission in this outbreak. The epidemic curve showing a gap of 
12-18 days between the single primary case and the secondary cases corresponds with the incubation period of human NiV infection [8]. Many of the index case's contacts $(43 \%)$, who came in physical contact with the index case when he was ill, subsequently became ill. In the case-control study, case-patients were significantly more likely to be in contact with the index case and were significantly more likely to be near him when he was coughing. As subsequent cases were limited to close contacts of the primary case, and none of the contacts of the subsequent cases developed illness, we conclude that the index case was the only $\mathrm{NiV}$ transmitter in this outbreak.

Five $(83 \%)$ of the subsequent cases came in contact with the index case only during the last 2 days of his illness when he developed respiratory symptoms. NiV has been isolated from human saliva, urine, nasal and pharyngeal secretions $[9,10]$ and there is evidence of spread of NiV infection from direct contact with respiratory secretions or other body secretions of infected pigs and humans $[2,11,12]$. The probability of NiV transmission is probably amplified during the last stages of illness when respiratory symptoms become more prominent and perhaps the concentration of virus in respiratory secretions increases. In Bangladesh, as the level of physical contact with the patient intensifies with the severity of the disease [13], this further increases the risk of transmission.

The NiV neutralizing antibody prevalence was $>50 \%$ in the bats sampled from the outbreak area which suggests that NiV has circulated in this population of bats. The result is consistent with findings in other pteropid bat populations in Malaysia, India, and Bangladesh [1, 5, 14, 15]. The bat survey was performed approximately 1 month after the onset of illness in the index case, and it is possible that infected bats were present in the colony around the time of the first human infection. Furthermore, the index case had no evidence of exposure to clinically ill domestic animals. He also had history of drinking raw date palm juice before his illness which has been associated with $\mathrm{NiV}$ infection in a previous outbreak investigation [3]. These lines of evidence suggest that the virus was probably transmitted directly from its natural reservoir, rather than an intermediate domestic animal.

A limitation of our study is its reliance on proxy interviews for some of the case-patients. This may have obscured some exposure information. However, we started our investigation within 14 days of the death of the index case, and collected information from several proxy respondents thus reducing the likelihood that we failed to collect information on probable exposure to risk factors. Another limitation is the lack of serological data from controls. There is evidence of subclinical infection of $\mathrm{NiV}$ from Malaysia [16] which could have reduced power to identify association due to erroneous inclusion of cases as controls. Even with this potential limitation our results identified a biologically credible pathway for transmission.

Findings from outbreaks in Siliguri and Faridpur illustrate that human-to-human transmission has occurred repeatedly in the Indian subcontinent. The social norm in Bangladesh is that family members and loved ones provide hands-on care to sick patients [13]. Further, hospital healthcare workers in Bangladesh are reluctant to provide hands-on care to admitted patients which increases the risk of transmission to family members and relatives who provide care without any training or supplies to reduce the risk of transmission [13]. Efforts to educate caregivers of their risk especially at later stages of illness, while maintaining sensitivity to cultural mores, and promoting basic infection control practices such as washing hands with soap after handling patients and avoiding close physical contact [2] could limit transmission of $\mathrm{NiV}$ and other diseases in people who care for sick patients.

\section{ACKNOWLEDGEMENTS}

The authors thank all the study participants for their contribution at a very difficult time. We gratefully acknowledge the relentless effort of the field officers involved in data collection and compilation and cooperation of the staff at Rangpur Medical College Hospital and Dinajpur Sadar Hospital. Our gratitude to the Civil Surgeon of Thakurgaon District and the Upazila Health and Family Planning Officer of Haripur subdistrict for their extensive cooperation with the investigation. We are also grateful to the Australian Animal Health Laboratory for their support with the animal investigation.

This work was funded by the Centers for Disease Control and Prevention (CDC), Atlanta, the U.S. National Institutes of Health, International Collaborations in Infectious Disease Research (ICIDR) Opportunity Pool, the Government of Bangladesh (GoB) through IHP-HNPRP, an NIH/NSF 'Ecology of Infectious Diseases' award from the John E. Fogarty International Center 2R01-TW005869, 
U.S. National Institutes of Health awards AI57158, AI070411, AI067549, the U.S. Department of Defense and Google.org. ICDDR,B acknowledges with gratitude the commitment of CDC, the U.S. National Institutes of Health, and the Government of Bangladesh to the Centre's research efforts.

\section{DECLARATION OF INTEREST}

None.

\section{REFERENCES}

1. Hsu VP, et al. Nipah virus encephalitis reemergence, Bangladesh. Emerging Infectious Diseases 2004; 10: 2082-2087.

2. Gurley ES, et al. Person-to-person transmission of Nipah virus in a Bangladeshi community. Emerging Infectious Diseases 2007; 13: 1031-1037.

3. Luby SP, et al. Foodborne transmission of Nipah virus, Bangladesh. Emerging Infectious Diseases 2006; 12: 1888-1894.

4. Montgomery J, et al. Risk factors for Nipah virus encephalitis in Bangladesh. Emerging Infectious Diseases 2008; 4: 1526-1532.

5. Epstein JH, et al. Henipavirus infection in fruit bats (Pteropus giganteus), India. Emerging Infectious Diseases 2008; 14: 1309-1311.

6. Chadha MS, et al. Nipah virus-associated encephalitis outbreak, Siliguri, India. Emerging Infectious Diseases 2006; 12: 235-240.
7. Daniels P, Ksiazek T, Eaton BT. Laboratory diagnosis of Nipah and Hendra virus infections. Microbes and Infection/Institut Pasteur 2001; 3: 289-295.

8. Harit AK, et al. Nipah/Hendra virus outbreak in Siliguri, West Bengal, India in 2001. Indian Journal of Medical Research 2006; 123: 553-560.

9. Harcourt BH, et al. Genetic characterization of Nipah virus, Bangladesh, 2004. Emerging Infectious Diseases 2005; 11: 1594-1597.

10. Goh KJ, et al. Clinical features of Nipah virus encephalitis among pig farmers in Malaysia. New England Journal of Medicine 2000; 342: 1229-1235.

11. Sahani M, et al. Nipah virus infection among abattoir workers in Malaysia, 1998-1999. International Journal of Epidemiology 2001; 30: 1017-1020.

12. Parashar UD, et al. Case-control study of risk factors for human infection with a new zoonotic paramyxovirus, Nipah virus, during a 1998-1999 outbreak of severe encephalitis in Malaysia. Journal of Infectious Diseases 2000; 181: 1755-1759.

13. Blum LS, Khan R, Nahar N. In-depth assessment of an outbreak of Nipah Encephalitis with person-to-person transmission in Bangladesh: implications for prevention and control strategies. American Journal of Tropical Medicine and Hygiene 2009; 80: 96-102.

14. Johara MY, et al. Nipah virus infection in bats (Order Chiroptera) in Peninsular Malaysia. Emerging Infectious Diseases 2001; 7 : 439-441.

15. Junsuke S, et al. Nipah virus survey of flying foxes in Malaysia. Japan Agricultural Research Quarterly 2007; 41: 69-78.

16. Chua KB. Nipah virus outbreak in Malaysia. Journal of Clinical Virology 2003; 26: 265-275. 